\title{
Building and hacking open source hardware
}

\author{
Simone Monachino $\$$, Eric James McDermottl, Andre Maia Chagas $₫$ \\ ‡ School of Computing, Newcastle University, Newcastle upon Tyne, United Kingdom \\ $\S$ Centre for Mind/Brain Science (CIMeC), University of Trento, Trento, Italy \\ | International Max Planck Research School, University of Tübingen, Tübingen, Germany \\ If School of Life Sciences, University of Sussex, Brighton, United Kingdom
}

Corresponding author: Andre Maia Chagas (andremaia.chagas@gmail.com)

Reviewable v1

Received: 16 Nov 2018| Published: 11 Dec 2018

Citation: Monachino S, McDermott E, Maia Chagas A (2018) Building and hacking open source hardware.

Research Ideas and Outcomes 4: e31701. https://doi.org/10.3897/rio.4.e31701

\section{Abstract}

The first edition of the Aspects of Neuroscience Brainhack took place at the Department of Physics at University of Warsaw, Poland between November 17th and 19th 2017. This hackathon was one of the satellite events of the Aspects of Neuroscience conference, it was organized by the Brainhack organization to promote interaction between researchers, encouraging open (neuro)science and collaborations on projects related to the study of the nervous system. The event had a total of nine projects on many different topics including functional connectivity research, white matter tractography, classification of brain-ageing biomarkers through machine learning, presentation of a portable one channel EEG registration device and a do it yourself 3D-printed neurobiology lab. The latter is highlighted in this paper.

\section{Keywords}

Neuroscience, Open science, Open source hardware, FlyPi, DIY science, Optical microscopy, Fluorescence, Optogenetics, Behaviour 


\section{Date and place}

17/11/2017-19/11/2017, University of Warsaw, Faculty of Physics, Pasteura 5, 02-093 Warsaw, Poland.

\section{List of participants}

Simone Monachino, Mateusz Kostecki, Karolina Rojek, Piotr Jedryszek, Michal Narbutt, Marcin Lipiec, Tomasz Lebitko, Tomasz Nikolaev, Ludwika Szczepanska, Kacper Kondrakiewicz.

Facilitators: André Maia Chagas, Eric James McDermott

\section{Introduction}

Neuroscience has a long history of experiments carried out with instruments built by researchers themselves (Boycott 1998, Matthews 2004, Zheng and Trudeau 2015), with the best-known example being perhaps the work of Alan Lloyd Hodgkin and Andrew Fielding Huxley on electrical conduction in the squid giant axon, which they carried out with a bioamplifier developed in the lab (Hodgkin and Huxley 1952). The experiments conducted with the device helped the understanding of neuronal communication and opened up an entire new study field in brain science.

In parallel to building their own tools, researchers also have the option of obtaining their tools from commercial sources. Although faster and "stress free", this path tends to pose as a barrier for a lot of labs and groups due to cost barriers and a general lack of availability of resources; this leads to a stunting of science and education potential (Maia Chagas 2018).

During the workshop students were exposed to this "do-it-yourself" tradition in order to become empowered with the confidence to make their own tools, take their research ideas into their own hands, as well as get familiar with the movement to make neuroscience more open and accessible.

Due to the limited time, a project-based approach was used. Participants were guided through the building steps of an affordable ( $€ 150$ / unit) open source neurobiology lab, "the FlyPi", (Chagas et al. 2017), which can be used to study the behavioural traits of many animal models currently used in brain sciences such as the nematode Caenorhabditis elegans or the arthropod Drosophila melanogaster. The FlyPi allows the ability to use several imaging techniques such as fluorescence imaging, calcium imaging, and highresolution movement tracking. The device also allows the modulation of neuronal activity using opto- and thermogenetics. The FlyPi can be easily built following online instructions (Chagas and Baden 2017). 


\section{Key outcomes and discussions}

Ten students and PhD candidates partook in the three-day project. The participants built the devices and performed several demo tests starting from the hardware and software components provided by the facilitators. The final apparatus, the $\mathrm{FlyPi}$, consists of a Raspberry $\mathrm{Pi}$ machine assembled in a 3D-printed structure, equipped with a highresolution camera for conducting imaging experiments. The portable lab also allows the implementation of other Arduino compatible modules and, therefore, the possibility to perform other neuroscience experimental paradigms such as optogenetics, fluorescence microscopy or behavioural tests. For example, during the project, optical and thermal components were added to the base design of the FlyPi by the participants. Throughout the course of the workshop, the young researchers had the opportunity to get hands-on experience with electronic components soldering during several "hacking" sessions. The project sessions were interspersed with scientific talks about open science and reproducible research. Two social events aimed to promote the integration and collaboration between the participants and the sharing of ideas and knowledge among the community were also organised as part of the hackathon. An ending presentation to summarise the activity performed during the project was finally conducted during the last day of the workshop.

\section{Conclusions}

The three-day event presented here provided the opportunity for young researchers in the field of neuroscience to build an open, affordable, customizable experimental setting device.

The FlyPi units that were built by the participants during the project were given to the young researchers, allowing them to freely modify the source code, customise the hardware components based on their scientific theoretical and experimental demands, and to perform automated investigations in accordance with the open science spirit of the workshop.

The project, as part of the AoN Brainhack Warsaw (Craddock et al. 2016), focused on open source software and hardware approaches to research with the intention to reduce the costs of present science and make research more reproducible, transparent and robust in the future.

\section{Acknowledgements}

"This project was carried out during AoN Brainhack Warsaw 2017. We would like to thank the organizers and IBRO for sponsoring equipment necessary to complete the project." 


\section{References}

- $\quad$ Boycott BB (1998) John Zachary Young, 18 March 1907-4 July 1997. Biographical Memoirs of Fellows of the Royal Society 44: 487-509. https://doi.org/10.1098/ rsbm.1998.0031

- $\quad$ Chagas AM, Baden T (2017) Flypi. 1.0.1. Release date: 2018-11-13. URL: https:// zenodo.org/record/1486176\#.W-sNfhCnzuQ

- Chagas AM, Prieto-Godino L, Arrenberg A, Baden T (2017) The $€ 100$ lab: A 3Dprintable open-source platform for fluorescence microscopy, optogenetics, and accurate temperature control during behaviour of zebrafish, Drosophila, and Caenorhabditis elegans. PLOS Biology 15 (7): e2002702. https://doi.org/10.1371/journal.pbio.2002702

- Craddock RC, Margulies D, Bellec P, Nichols BN, Alcauter S, Barrios F, Burnod Y, Cannistraci C, Cohen-Adad J, Leener BD, Dery S, Downar J, Dunlop K, Franco A, Froehlich CS, Gerber A, Ghosh S, Grabowski T, Hill S, Heinsfeld AS, Hutchison RM, Kundu P, Laird A, Liew S, Lurie D, McLaren D, Meneguzzi F, Mennes M, Mesmoudi S, O'Connor D, Pasaye E, Peltier S, Poline J, Prasad G, Pereira RF, Quirion P, Rokem A, Saad Z, Shi Y, Strother S, Toro R, Uddin L, Van Horn J, Van Meter J, Welsh R, Xu T (2016) Brainhack: a collaborative workshop for the open neuroscience community. GigaScience 5 (1). https://doi.org/10.1186/s13742-016-0121-x

- Hodgkin AL, Huxley AF (1952) A quantitative description of membrane current and its application to conduction and excitation in nerve. The Journal of Physiology 117 (4): 500-544. https://doi.org/10.1113/iphysiol.1952.sp004764

- Maia Chagas A (2018) Haves and have nots must find a better way: The case for open scientific hardware. PLOS Biology 16 (9): e3000014. https://doi.org/10.1371/ journal.pbio.3000014

- Matthews PC (2004) Historical analysis of the neural control of movement from the bedrock of animal experimentation to human studies. Journal of Applied Physiology 96 (4): 1478-1485. https://doi.org/10.1152/japplphysiol.00978.2003

- $\quad$ Zheng J, Trudeau MC (2015) Handbook of Ion Channels. CRC Press, Boca Raton, 691 pp. https://doi.org/10.1201/b18027 